\title{
MODEL BASED AUTOMATIC SEGMENTATION OF TREE STEMS FROM SINGLE SCAN DATA
}

\author{
Ruedi Boesch \\ Swiss Federal Institute for Forest, Snow and Landscape Research WSL \\ CH-8903 Birmensdorf, Switzerland \\ ruedi.boesch@wsl.ch
}

KEY WORDS: forestry, point cloud, RANSAC, segmentation, terrestrial laser scanning, tree detection

\begin{abstract}
:
Forest inventories collect feature data manually on terrestrial field plots. Measuring large amounts of breast height diameters and tree positions is time consuming. Terrestrial laser scanning could be an additional instrument to collect precise and full inventory data in the 3D space. As a preliminary assumption single scan data is used to evaluate a minimal data acquisition scheme. To extract features like trees and diameter from the scanned point cloud, a simple geometric model world is defined in 3D. Trees are cylinder shapes vertically located on a plane. Using a RANSAC-based segmentation approach, cylinders are fitted iteratively in the point cloud. Several threshold parameters increase the robustness of the segmentation model and extract point clouds of single trees, which still contain branches and the tree crown. Fitting circles along the stem using point cloud slices allows to refine the effective diameter for customized heights. The cross section of a single tree point cloud covers only the semi circle towards the scan location, but is still contiguous enough to estimate diameters by using a robust circle fitting method.
\end{abstract}

\section{INTRODUCTION}

Terrestrial laser scanning (TLS) can be used to collect efficiently three-dimensional measurements on forest inventory plots. The automatic extraction of structural features in a forest environment is still an algorithmic challenge. Many successful approaches exist for man-made structures like roads and roofs, mainly using aerial laser scanning (Bitelli et al., 2012; Lehtomaki et al., 2010).

In environmental research applications TLS data is used to extract soft features like slope, river beds or shrub canopies. Spatial statistics for topographic patterns (Brasington, Vericat et al. 2012) or spatial wavelets (Vierling et al., 2012) allow to quantify surface changes or characteristics from point clouds, but are not appropriate to extract inventory related feature measurements.

Forest inventories require detailed ground reference data of stems and tree coverage, which are typically collected manually within a field plot, but the achieved accuracy of biomass estimation on the local plot level is poor (Kankare et al., 2013). Additional TLS data collection has the potential to improve accuracy and efficiency of field inventory and to provide appropriate features like full coverage of tree positions and volume estimation. A combined terrestrial field plot with manual and automatically acquired features raises the question, what kind of scanning effort is needed to extract robust forest inventory related features. The acquisition and registration of multiple scans for a single plot is rather time and resource consuming, therefore the aim of this study is to evaluate only single scans initially.

In this paper a simple model world for feature extraction of single trees is used (Liang et al., 2012; Ling and Jie, 2008). Trees are defined as cylinder shapes, which are vertically located on a plane surface in the 3D space (Fig. 1).

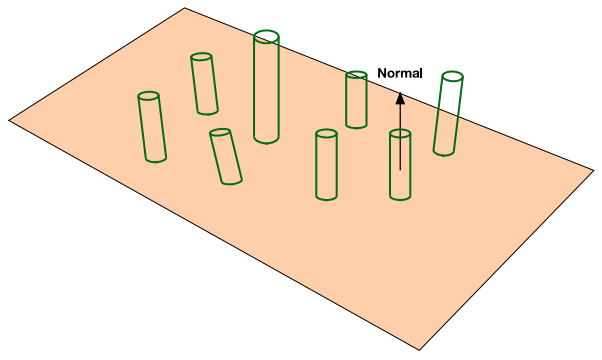

Figure 1. cylinder world

Similar model definitions are very common in robotics and computer vision, but have also already been applied successfully by different forestry oriented applications (Lari et al., 2011; Lehtomaki et al., 2010; Raumonen et al., 2011). More sophisticated shape models are mainly used with airborne laser scanning (Jochem et al., 2011; Pfeifer and Briese, 2007).

Many contributions require a digital terrain model (DTM), which implicitly contain a robust estimate of the surface (Henning and Radtke, 2006). In this study only TLS data has been used to derive a surface plane. Often a DTM with a corresponding accuracy or acquisition time is not available. Therefore the fitting performance depending only on TLS data is one of the major research question in this study.

After defining the data model, a suitable extraction process has to be discussed. 3D feature extraction can be characterized by access methods and neighbourhood representations (Pirotti et al., 2012). Currently several different method categories exist like voxel-based clustering, model fitting and region growing, but obviously there is a considerable conceptual overlap within them. 


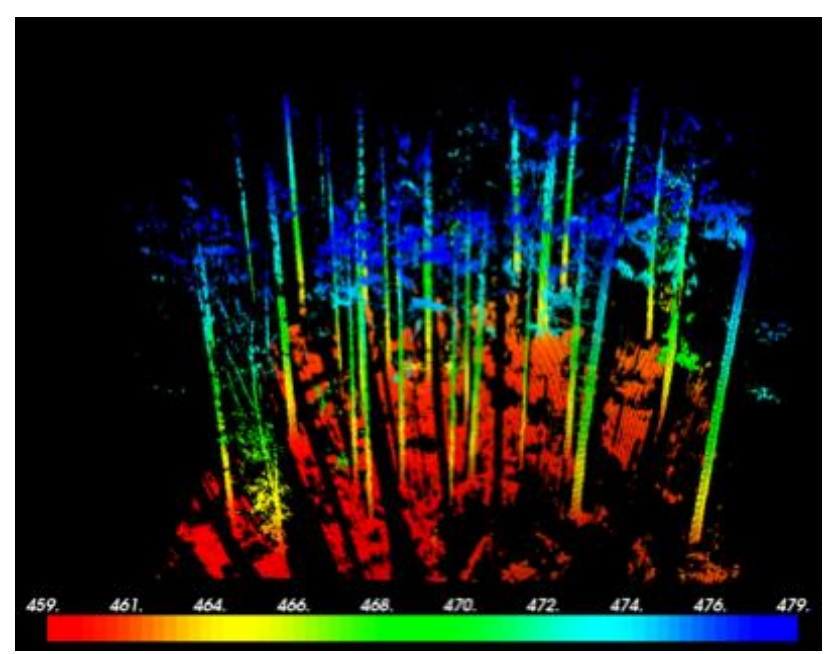

Figure 2. single scan study area "Bremgarten", colors in m a.s.l

Voxel-based approaches are 3D extended raster algorithms and widely used due the simple data model and many proven algorithms exist like marching cubes or clustering (Gorte and Pfeifer, 2004; Henning and Radtke, 2006; Maas et al., 2008). Segmentation of 3D point clouds identifies similar points with a model-based approach. For non-rigid shapes like canopies, shrub or tree stems, the robustness of model fitting becomes crucial. Region growing identifies in a first step seed surfaces consisting of a group of nearby points (Vosselman et al., 2004). Then seed surfaces are growing with further points if the perpendicular distance is below a threshold. Finding a significant seed surface in noisy vegetation data is crucial and similar to model fitting initially.

\section{MATERIAL AND METHODS}

\subsection{Study Area}

The study area "Bremgarten" is characterized by a moderate mixed deciduous forest with beeches, ashes and oaks.

\begin{tabular}{|l|l|}
\hline Scanner & Leica ScanStation C10 \\
\hline Date & 26. September 2012 \\
\hline Wavelength & $532 \mathrm{~nm}$ \\
\hline Beam diameter & $1 \mathrm{~cm}$ at $20 \mathrm{~m}$ \\
\hline Raw data points & 55.4 million points \\
\hline
\end{tabular}

Table 1. Scan configuration

The dataset has been reduced to 2.7 million points with ca. $6 \mathrm{~cm}$ angular resolution, using the software Leica Cylone ("medium reduction") (Fig. 2)

\subsection{Methods}

The main focus in this paper is on robust and still efficient model fitting. Scanning vegetation areas with a terrestrial laser scanner requires to define a multi- or single-scan data acquisition. Both schemes require coregistration with reference targets and survey registration points. In a single-scan measurement, the scanner is placed in the plot center and a full field of view scan is executed.
Several publications try to reduce the computational effort and ambiguity problems by using $2 \mathrm{D}$ search methods with circle fitting (Maas et al., 2008). Due to inherent reflection in the direction of a laser beam, the backside of every object is partially missing in the resulting dataset. Therefore contain vertical slices of a single scan point cloud only semi circles, which are often distorted due to occlusion by closer trees (Fig 3 and 7).

Further occluding branches and bushes generate many 3D outliers, which cannot be filtered without detailed knowledge about object structure. Multi-scans can be used to improve point cloud density and accuracy by merging, but automatic coregistration is not available and a high acquisition effort is needed. In order to evaluate acquisitions with minimal effort, only single scan datasets have been used.

A well-known algorithm to address these problems is the random sample consensus (RANSAC)(Fischler and Bolles, 1981). This algorithm is used in many applications as fitting principle (Cho et al., 2012; Hyyppa et al., 2001) and robustness and efficiency is addressed as well (Elseberg et al., 2013).

The aim of the study is to evaluate robust and still efficient extraction methods for point clouds in a typical commercial forest. The software framework Point Cloud Library (PCL) (Rusu and Cousins, 2011) has been used, mainly because of its mature status and very active development.

\section{RESULTS}

In the first processing step, surface normals of the point cloud are estimated. The calculated vectors allow the fitting of a surface plane, corresponding to the forest floor in our simplified model world. After eliminating the surface plane points from the point cloud an iterative search for single cylinders is implemented as follows.

PCL defines generic shape primitives like plane, circle, cone, torus or cylinder. For a cylinder fitting model 7 parameters are required:

- 3D point on its axis

- 3D axis direction

- radius

To increase the fitting robustness, additional thresholds for a valid point distance, radius and surface normal weight can be defined, which determine how close a point must be to the cylinder model in order to be considered as valid inlier. This approach achieves a preliminary discrete outlier elimination, where RANSAC removes the remaining outliers by shape fitting (Fig 3).

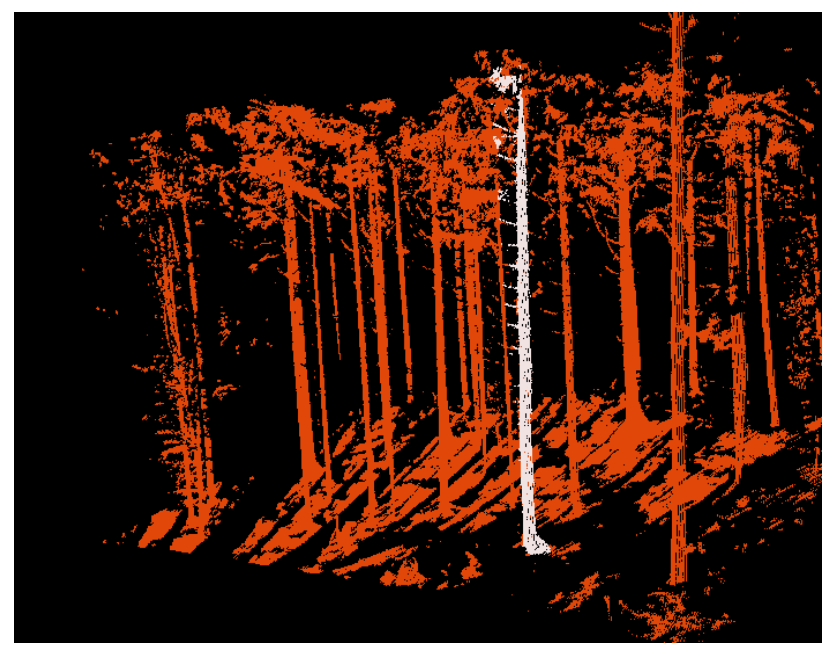

Figure 3. cylinder fitting of first tree 
If the radius tolerance is too big, the axis direction will be tilted artificially due to the wrong cylinder fitting (right sample of Fig. 4)

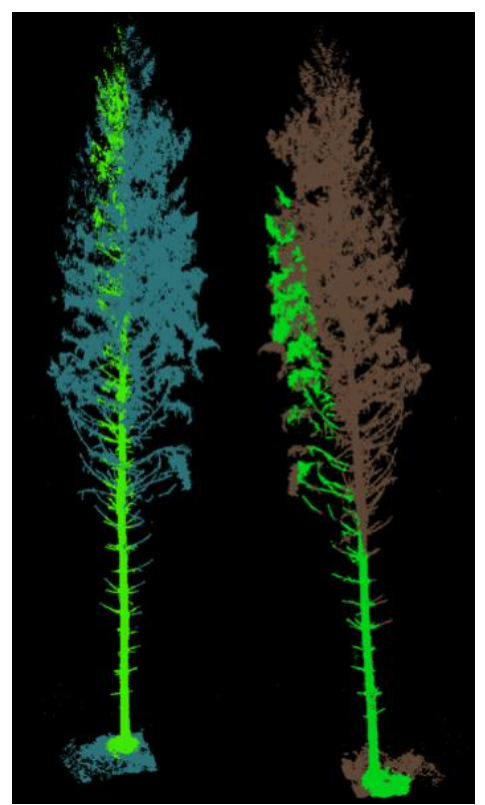

Fig 4. RANSAC cylinder fitting (green) with radius tolerance $1 \mathrm{~m}$ (left) and $2 \mathrm{~m}$ (right)

The search for further cylinders continues until the radius of the matched cylinder is below a threshold. Because trees are not perfectly vertical, an additional Z-axis tolerance threshold has been used, which defines the allowed deviation from a perfect vertical unit vector $[0,0,1]$ (Fig. 5).

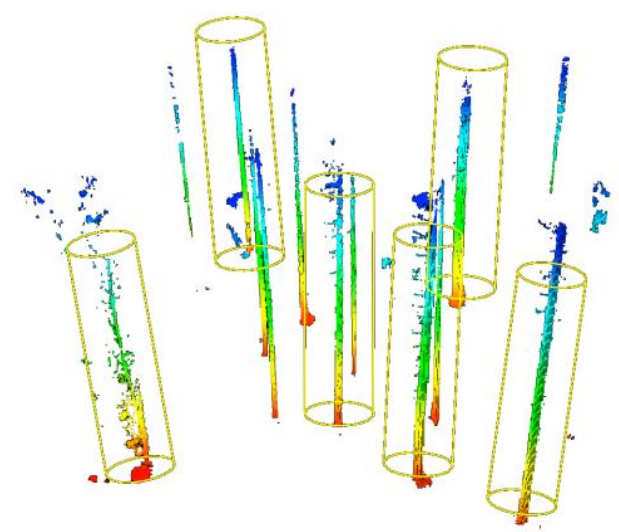

Fig 5. Extracted tree clouds from the study area (cylinders indicate estimated Z-axis direction)

In the current implementation the following thresholds have been used (Table 2).

\begin{tabular}{|l|l|}
\hline Minimal radius & $0.1 \mathrm{~m}$ \\
\hline Maximal radius & $1.0 \mathrm{~m}$ \\
\hline Point distance & $0.1 \mathrm{~m}$ \\
\hline Normal weight & 0.2 \\
\hline Z-axis direction tolerance & 0.95 (rel. to unit vector 1 ) \\
\hline
\end{tabular}

Table 2. Segmentation parameters for cylinder fitting

After single tree cylinders are extracted, the radius of each stem represents a global estimate, but a more detailed approximation is required to extract the effective diameter at specified heights of a single tree. The cylinder radius is often overestimated due to the fitting with branches and tree crown (Fig 4).

Therefore an additional fitting process along the tree axis has been applied using horizontal slices of the single tree point cloud. The position of the slices is defined by the vertical position of the reference diameter measurements. The thickness of each slice has been empirically chosen as $0.4 \mathrm{~m}$ (Fig 6).

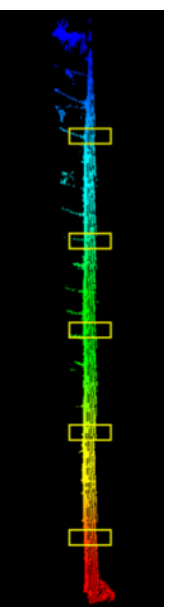

Fig 6. Slices for local diameter estimation

To achieve a robust fit the circle segments are fitted in the 2D space using the Eigenvector approach by (Taubin, 1991). This allows to estimate tree diameters for coarse single scan datasets (Fig 7).
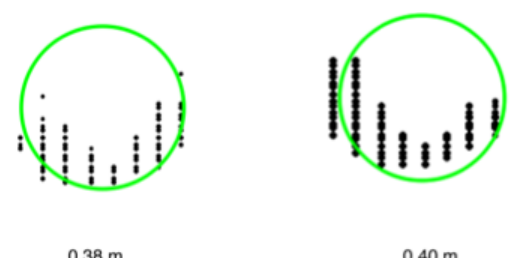

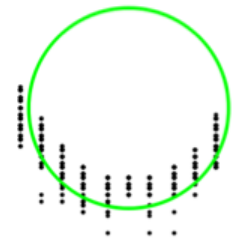

$0.48 \mathrm{~m}$

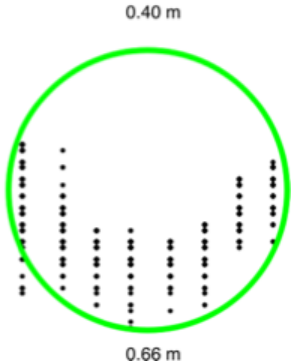

$0.66 \mathrm{~m}$
Fig 7. Cross sections (Z 4.8 - 5.2 m) of 4 extracted tree clouds (black) with fitted tree diameters (green) 
To verify the fitting accuracy, a further comparison with a full $360^{\circ}$ scan of a single reference tree has been made. Reference diameters at different heights have been measured after cutting the tree by a forester (Fig. 8).

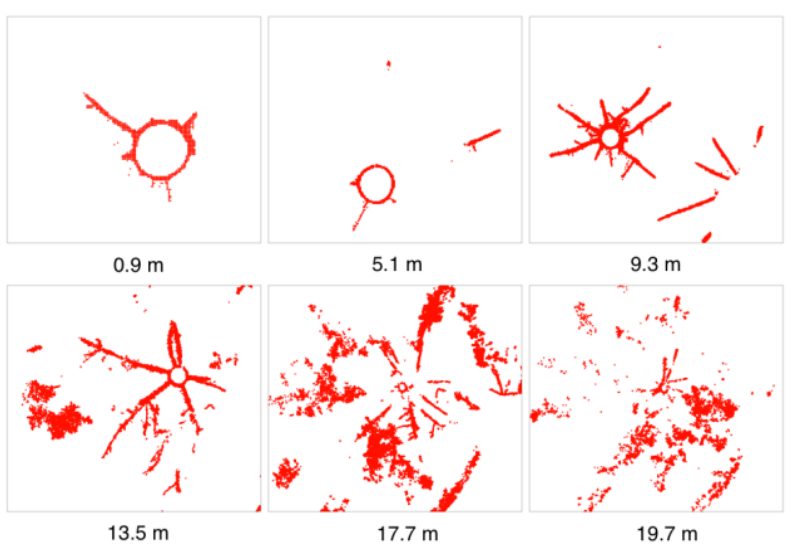

Fig 8. Cross section slices $(0.4 \mathrm{~m})$

Fitting a circle in the cross section slices reveals a minor underestimation of the automatically calculated diameters (Fig. 9, Tab. 3). Above $15 \mathrm{~m}$ the matching process fails, because the circle of the stem could not be detected (Fig. 8, 17.7m) or no circular structure can be recognized (Fig. 8, 19.7m).

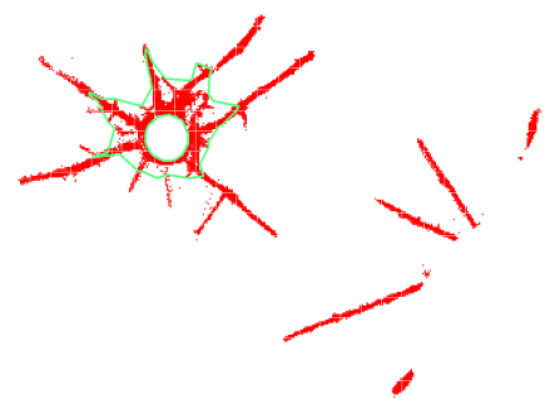

Fig 9. Cross section (9.3m) with fitting circle (green)

\begin{tabular}{|c|c|c|c|}
\hline$Z[\mathrm{~m}]$ & Model Fitted & Manual & Error $[\mathrm{cm}]$ \\
\hline 0.9 & 32 & 38 & -6 \\
\hline 5.1 & 29 & 31 & -2 \\
\hline 9.3 & 27 & 28 & -1 \\
\hline 13.5 & 22 & 25 & -3 \\
\hline 17.7 & - & 21 & - \\
\hline 19.7 & - & 19 & - \\
\hline
\end{tabular}

Table 3. Diameter comparison of a single tree slices

\section{CONCLUSIONS}

RANSAC-based fitting of cylinder models with sparse point clouds is robust enough to detect single trees and corresponding diameters with a reduced field acquisition effort. Single scan data can be used to gain additional forest inventory parameters for better calibration of biomass and forest stand models.

Finding cylinders on a plane is a suitable model view, but many forest stands are far from an ideal flat plane. Bootstrapping with a surface is crucial to get an initial orientation of the scene, but is also sensitive to appropriate parameter selection. Therefore robust estimates of characteristic parameters (forest stand type, species distribution, age) from a larger area than the local plot are required.

If branches are part of the fitted cylinder, the estimated diameter will be overestimated. This contradiction cannot be solved with the simple topology of the model.

Tilted trees and terrain slope may be handled more appropriate by random partitioning of the initial point cloud, if statistical features of a low resolution DTM is used. The fitting process may improved by adopting a cone instead of a cylinder as basic tree model.

\section{REFERENCES}

Bitelli, G., Simone, A., Girardi, F., Lantieri, C., 2012. Laser Scanning on Road Pavements: A New Approach for Characterizing Surface Texture. Sensors-Basel 12, 9110-9128.

Cho, K., Baeg, S.H., Park, S., 2012. Multiple Object Detection and Classification on Uneven Terrain using Multi-Channel LIDAR for UGV. Unmanned Systems Technology Xiv 8387.

Elseberg, J., Borrmann, D., Nuchter, A., 2013. One billion points in the cloud - an octree for efficient processing of $3 D$ laser scans. Isprs J Photogramm 76, 76-88.

Fischler, M.A., Bolles, R.C., 1981. Random Sample Consensus a Paradigm for Model-Fitting with Applications to ImageAnalysis and Automated Cartography. Commun Acm 24, 381395.

Gorte, B., Pfeifer, N., 2004. Structuring laser-scanned trees using $3 D$ mathematical morphology, XXth ISPRS Congress. ISPRS, Istanbul, Turkey, pp. 929-933.

Henning, J.G., Radtke, P.J., 2006. Detailed stem measurements of standing trees from ground-based scanning lidar. Forest Sci 52, 67-80.

Hyyppa, J., Kelle, O., Lehikoinen, M., Inkinen, M., 2001. A segmentation-based method to retrieve stem volume estimates from 3-D tree height models produced by laser scanners. Ieee Transactions on Geoscience and Remote Sensing 39, 969-975.

Jochem, A., Hollaus, M., Rutzinger, M., Hofle, B., 2011. Estimation of Aboveground Biomass in Alpine Forests: A SemiEmpirical Approach Considering Canopy Transparency Derived from Airborne LiDAR Data. Sensors-Basel 11, 278295.

Kankare, V., Holopainen, M., Vastaranta, M., Puttonen, E., Yu, X.W., Hyyppa, J., Vaaja, M., Нyyppa, H., Alho, P., 2013. Individual tree biomass estimation using terrestrial laser scanning. Isprs J Photogramm 75, 64-75. 
Lari, Z., Habib, A.F., Kwak, E., 2011. An adaptive approach for segmentation of $3 d$ laser point cloud, Laserscanning 2011 Workshop. ISPRS, Calgary, Canada, p. 6.

Lehtomaki, M., Jaakkola, A., Hyyppa, J., Kukko, A., Kaartinen, H., 2010. Detection of Vertical Pole-Like Objects in a Road Environment Using Vehicle-Based Laser Scanning Data. Remote Sens-Basel 2, 641-664.

Liang, X.L., Litkey, P., Hyyppa, J., Kaartinen, H., Vastaranta, M., Holopainen, M., 2012. Automatic Stem Mapping Using Single-Scan Terrestrial Laser Scanning. Ieee Transactions on Geoscience and Remote Sensing 50, 661-670.

Ling, Z., Jie, Z., 2008. Obtaining Three-dimensional Forest Canopy Structure Using TLS. Remote Sensing and Modeling of Ecosystems for Sustainability V 7083.

Maas, H.G., Bienert, A., Scheller, S., Keane, E., 2008. Automatic forest inventory parameter determination from terrestrial laser scanner data. International Journal of Remote Sensing 29, 1579-1593.

Pfeifer, N., Briese, C., 2007. ISPRS Workshop on Laser Scanning 2007 and SilviLaser 2007, Espoo, Finland, pp. 311320.

Pirotti, F., Grigolato, S., Lingua, E., Sitzia, T., Tarolli, P., 2012. Laser Scanner Applications in Forest and Environmental Sciences. Ital J Remote Sens 44, 109-123.

Raumonen, P., Kaasalainen, S., Kaasalainen, M., Kaartinen, H., 2011. Approximation of volume and branch size distribution of trees from laser scanner data, Laserscanning 2011 Workshop. ISPRS, Calgary, Canada, p. 6.

Rusu, R.B., Cousins, S., 2011. 3D is here: Point Cloud Library (PCL), IEEE International Conference on Robotics and Automation (ICRA), Shanghai, China, p. 5.

Taubin, G., 1991. Estimation of Planar Curves, Surfaces, and Nonplanar Space-Curves Defined by Implicit Equations with Applications to Edge and Range Image Segmentation. Ieee Transactions on Pattern Analysis and Machine Intelligence 13, 1115-1138.

Vierling, L.A., Xu, Y.Y., Eitel, J.U.H., Oldow, J.S., 2012. Shrub characterization using terrestrial laser scanning and implications for airborne LiDAR assessment. Can J Remote Sens 38, 709-722.

Vosselman, G., Gorte, B., Sithole, G., Rabbani, T., 2004. Recognising structure in laser scanner point clouds. ISPRS, Freiburg, Germany, pp. 33-38. 